\title{
First-line pembrolizumab/placebo plus trastuzumab and chemotherapy in HER2-positive advanced gastric cancer: KEYNOTE-811
}

\author{
Hyun Cheol Chung*,1 (D), Yung-Jue Bang², Charles S Fuchs ${ }^{3}$, Shu-Kui Qin ${ }^{4}$, Taroh Satoh ${ }^{5}$, \\ Kohei Shitara ${ }^{6}$, Josep Tabernero7, Eric van Cutsem ${ }^{8}$, Maria Alsina7, Zhu Alexander Cao ${ }^{9}$, Jia \\ Lu $^{9}$, Pooja Bhagia ${ }^{9}$, Chie-Schin Shih ${ }^{9} \&$ Yelena Y Janjigian ${ }^{10}$ \\ ${ }^{1}$ Division of Medical Oncology, Yonsei Cancer Center, Yonsei University College of Medicine, Seoul, 03722, South Korea \\ ${ }^{2}$ Seoul National University College of Medicine, Seoul, 03080, South Korea \\ ${ }^{3}$ Yale Cancer Center, Smilow Cancer Hospital, New Haven, CT 06510, USA \\ ${ }^{4}$ Cancer Center of People's Liberation Army, Nanjing, 210002, China \\ ${ }^{5}$ Osaka University, Suita, Osaka, 565-0871, Japan \\ ${ }^{6}$ National Cancer Center Hospital East, Kashiwa, 277-8577, Japan \\ ${ }^{7}$ Vall d'Hebron University Hospital \& Institute of Oncology, Barcelona, 08035, Spain \\ ${ }^{8}$ University Hospitals Gasthuisberg Leuven \& KU Leuven, Leuven, 03001, Belgium \\ ${ }^{9}$ Department of Medical Oncology, Merck \& Co., Inc., Kenilworth, NJ 07033, USA \\ ${ }^{10}$ Memorial Sloan Kettering Cancer Center, New York, NY 10065, USA \\ *Author for correspondence: unchung8@yuhs.ac
}

Treatment options for patients with HER2-positive advanced gastric cancer are limited, and the prognosis for these patients is poor. Pembrolizumab has demonstrated promising antitumor activity in patients with advanced gastric or gastroesophageal junction adenocarcinoma as monotherapy, in combination with chemotherapy and in combination with trastuzumab. Combining pembrolizumab with trastuzumab and chemotherapy may therefore provide a benefit for patients with advanced HER2-positive gastric cancer. Here we aimed to describe the design of and rationale for the randomized, double-blind, placebocontrolled Phase III KEYNOTE-811 study, which will evaluate the efficacy and safety of pembrolizumab or placebo in combination with trastuzumab and chemotherapy as first-line treatment for patients with advanced HER2-positive gastric or gastroesophageal junction adenocarcinoma.

Clinical Trial Registration: NCT03615326 (ClinicalTrials.gov)

First draft submitted: 21 July 2020; Accepted for publication: 28 September 2020; Published online: 10 November 2020

Keywords: chemotherapy $\bullet$ first-line therapy $\bullet$ gastric cancer $\bullet$ gastroesophageal junction cancer $\bullet$ human epidermal growth factor receptor $2 \bullet$ pembrolizumab • trastuzumab

Gastric cancer is the fifth most common cancer in the world and the third-leading cause of cancer-related death [1]. More than 1 million new cases were diagnosed in 2018, and 780,000 people died of the disease. The incidence of gastric cancer is substantially higher in Eastern Asia than in any other region, and it is diagnosed twice as frequently in men. Although often considered a single entity, gastric cancers can be divided into noncardia gastric cancer, arising from the distal portion of the stomach, and cardia gastric cancer, which includes cancers of the upper stomach and the gastroesophageal junction (GEJ) [2]. The primary risk factor for the development of noncardia gastric cancer is the presence of Helicobacter pylori, which is believed to be causative in almost $90 \%$ of cases [3]. In contrast, cardia gastric cancers are more frequently associated with obesity and gastroesophageal reflux disease [2].

In addition to their topographic categorization, gastric cancers can be divided into one of four molecular subtypes, each associated with specific genomic and prognostic characteristics $[4,5]$. Of note, this molecular profiling has enabled the identification of potentially targetable alterations in gastric cancer and provides an opportunity to identify biomarkers of response. The most common of these subtypes is the chromosomally unstable tumors

Future Medicine 
(CIN) subtype, which accounts for approximately $50 \%$ of all gastric cancers [4]. This subtype is associated with intestinal type histology, elevated frequency in GEJ/cardia gastric cancer, TP53 mutation and amplification of receptor tyrosine kinases, including HER2 [4,6]. Consequently, HER2 overexpression is observed in 10-22\% of all gastric cancers $[6,7]$.

Surgical resection can be curative for patients with early-stage gastric cancer; however, because of the largely asymptomatic nature and aggressiveness of the disease at this stage, most diagnoses are made when the disease is advanced $[7,8]$. The prognosis for patients with advanced-stage gastric cancer is poor, with survival ranging from approximately 4 months with best supportive care to 12 months with chemotherapy [8]. The recommended first-line treatment for most patients with advanced gastric cancer is doublet combination therapy with platinum and fluoropyrimidine [7,9]. For patients with HER2-postive disease, the recommended first-line regimen is trastuzumab (anti-HER2) in combination with platinum and fluoropyrimidine-based chemotherapy [7,9]. The inclusion of trastuzumab in the treatment regimen for gastric cancer was based on the results of the Phase III ToGA trial (ClinicalTrials.gov: NCT01041404), which showed that the addition of trastuzumab to a regimen of capecitabine + cisplatin or fluorouracil + cisplatin improved overall survival (OS) from 11.1 to 13.8 months (hazard ratio [HR], 0.74; 95\% CI: $0.60-0.91 ; \mathrm{p}=0.0048$ ) and progression-free survival (PFS) from 5.5 to 6.7 months (HR: $0.71 ; 95 \%$ CI: $0.59-0.85 ; \mathrm{p}=0.0002$ ) in patients with HER2-positive gastric or GEJ cancer [10]. First-line treatment combinations with pertuzumab (anti-HER2) plus trastuzumab plus chemotherapy as well as lapatinib (tyrosine kinase inhibitor) plus chemotherapy versus placebo plus chemotherapy have not proven successful in Phase III trials thus far because of their failure to meet the primary end point in the JACOB and TRIO-013/LOGiC trials, respectively [11,12].

The choice of second-line or later therapy for patients with advanced gastric cancer is dependent on therapy previously received and on performance status [9]. The continuation of HER2 inhibitors after disease progression with trastuzumab has not been shown to be effective in prospective trials [13]. Recommended chemotherapy-based regimens include docetaxel, paclitaxel or irinotecan monotherapy; fluorouracil + irinotecan; and trifluridine and tipiracil (third-line or later therapy) [9]. The anti-vascular endothelial growth factor receptor 2 antibody ramucirumab in combination with paclitaxel is also recommended for use as second-line therapy based on the results of the Phase III RAINBOW trial (NCT01170663), which demonstrated improved survival with this combination compared with paclitaxel alone (median OS: 9.6 vs 7.4 months; HR: 0.807; 95\% CI: 0.678-0.962; $\mathrm{p}=0.017$ ) [9,14]. Pembrolizumab, an anti-PD-1 monoclonal antibody, is recommended by the National Comprehensive Cancer Network as the preferred second-line therapy for patients with microsatellite instability-high (MSI-H) or mismatch repair deficient tumors and as a third-line therapy for patients with PD-L1-positive (combined positive score [CPS] $\geq 1$ ) tumors [9]. The anti-PD-1 monoclonal antibody nivolumab is recommended by the Japanese Gastric Cancer Association as third-line therapy [15]. Of note, PD-1/PD-L1 inhibitors have not yet been approved by the EMA for use in patients with gastric cancer.

Given the limited survival benefit observed with currently recommended therapies, there remains a need for novel therapeutic regimens for the treatment of patients with advanced gastric cancer.

\section{KEYNOTE-811 trial}

Here we describe the design and rationale of the randomized, double-blind, placebo-controlled, Phase III KEYNOTE-811 study (NCT03615326), which will evaluate the efficacy and safety of pembrolizumab or placebo in combination with trastuzumab and chemotherapy as first-line treatment for patients with advanced HER2-positive gastric or GEJ adenocarcinoma.

\section{Background \& rationale}

The PD-1/PD-L1 pathway is an important regulatory component of the immune response and plays a critical role in tumor evasion of immune surveillance [16]. A growing body of evidence indicates that PD-1 and PD-L1 are frequently overexpressed in gastric cancer and that their upregulation may be prognostic of poor outcome [17,18]. Consequently, PD-1 and PD-L1 represent promising targets for the treatment of gastric cancer.

Pembrolizumab is a highly selective, humanized, monoclonal immunoglobulin G4-K antibody that binds to PD-1 and blocks its interactions with PD-L1 and PD-L2, thereby releasing PD-1 pathway-mediated inhibition of the antitumor immune response [19]. Pembrolizumab has demonstrated durable antitumor activity in patients with gastric cancer in several trials. In the Phase Ib KEYNOTE-012 study (NCT01848834), eight of 36 evaluable 
patients in the gastric cancer cohort achieved partial response (PR) with pembrolizumab monotherapy, for an objective response rate (ORR) of 22\% (95\% CI: 10-39) [20]. A clinically meaningful result was also observed in cohort 1 of the Phase II KEYNOTE-059 study (NCT02335411), which investigated the efficacy of third-line or later pembrolizumab monotherapy in patients with gastric or GEJ cancer [21]. Of 259 patients enrolled in this cohort, 30 patients (12\%; 95\% CI: $8-16)$ experienced objective response; six of those patients (2\%; 95\% CI: $1-5)$ achieved complete response (CR). The median OS in this cohort was 5.6 months. The potential benefit of pembrolizumab for the treatment of gastric cancer was also investigated in the Phase III KEYNOTE-061 trial (NCT02370498), which evaluated the efficacy of pembrolizumab monotherapy compared with paclitaxel in patients with advanced gastric or GEJ cancer that had progressed on first-line chemotherapy with platinum and fluoropyrimidine [22]. After follow-up of 7.9 months, the median OS in patients with CPS $\geq 1$ tumors -9.1 months for pembrolizumab and 8.3 months for paclitaxel (HR: 0.82; 95\% CI: 0.66-1.03; one-sided p = 0.0421) was not significantly different between the groups. PFS and ORR were similar between the treatment groups, but responses were more durable in patients who received pembrolizumab than those who received paclitaxel (median duration of response [DOR]: 18.0 vs 5.2 months, respectively). Post hoc analyses of patients with CPS $\geq 10$ tumors revealed a greater survival benefit for the pembrolizumab group than the paclitaxel group (HR: 0.64; 95\% CI: 0.41-1.02) [23]. In the Phase III KEYNOTE-062 study (NCT02494583), first-line pembrolizumab or pembrolizumab + chemotherapy versus chemotherapy was evaluated in patients with CPS $\geq 1$, HER2-negative, advanced gastric cancer [24]. Pembrolizumab monotherapy was noninferior to chemotherapy for OS in patients with CPS $\geq 1$ tumors but resulted in a clinically meaningfully improvement for OS in patients with CPS $\geq 10$ tumors (HR: 0.69; 95\% CI: 0.49-0.97). Pembrolizumab + chemotherapy did not show superior OS and PFS in patients with CPS $\geq 1$ tumors and OS in patients with CPS $\geq 10$ tumors. The KEYNOTE-059 study included two additional gastric cancer cohorts in its investigation of pembrolizumab as monotherapy or in combination with chemotherapy in previously untreated patients [25]. The ORR among patients who received first-line pembrolizumab monotherapy was $26 \%$ (95\% CI: 12-45; eight of 31 patients; 2 CR), and the median OS was 20.7 months. In patients who received first-line pembrolizumab in combination with a fluoropyrimidine and a cisplatin, the ORR was $60 \%$ (95\% CI: 39-79; 15 of 25 patients; 1 CR) and the median OS was 13.8 months.

These data demonstrate that pembrolizumab has durable clinical benefit in patients with advanced gastric cancer and shows promise when used in combination with chemotherapy. However, for patients with HER2-positive disease, the current standard of care also includes treatment with the HER2-targeted antibody trastuzumab [7,9]. In recent years, there has been growing interest in the use of trastuzumab in combination with immune checkpoint inhibitors because of observations that the immune system substantially contributes to the therapeutic effects of HER2-targeted antibodies and that treatment with trastuzumab may increase tumor expression of PD-L1 [26,27]. The combination of trastuzumab and pembrolizumab was initially investigated in the Phase Ib/II PANACEA trial (NCT02129556) in patients with trastuzumab-resistant advanced HER2-positive breast cancer [28]. Results indicated that the combination had manageable safety and durable clinical benefit, but only in patients with PD-L1-positive (CPS $\geq 1$ ) tumors. This study established proof of concept for this dual antibody approach, but the efficacy of these agents in combination with chemotherapy has not yet been established. Two early-phase trials (NCT02954536 and NCT02901301) are underway to investigate the combination of pembrolizumab, trastuzumab and chemotherapy for the treatment of gastric or GEJ cancer; both studies enrolled patients regardless of PD-L1 status. Additionally, these studies were not powered to distinguish differences based on PD-L1 status, although this was explored in NCT02954536; the authors suggested that the effect was similar regardless of PD-L1 status [29]. Results from one of these trials (NCT02954536) showed that a combination of pembrolizumab, trastuzumab and chemotherapy (oxaliplatin or cisplatin) had a manageable safety profile and promising efficacy, with an ORR of 91\% (95\% CI: 78-97; 32 of 35 patients; six CRs) in patients with esophagogastric adenocarcinoma [30]. In another trial (NCT02901301), the ORR was 77\% with pembrolizumab, trastuzumab, capecitabine and cisplatin as first-line therapy for HER-positive advanced gastric cancer [31]; the dosing schedule and patient population are similar to those of the KEYNOTE-811 trial.

Taken together, the available evidence indicates that pembrolizumab has durable efficacy in gastric cancer and that pembrolizumab combined with trastuzumab and chemotherapy is a promising treatment option in patients with HER2-positive disease. 
(A)

Key eligibility criteria
- Histologically or cytologically confirmed
diagnosis of previously untreated,
locally advanced unresectable or
metastatic HER2-positive gastric or
GEJ adenocarcinoma
- Measurable disease per RECIST v1.1
- ECOG performance status of 0 or 1
- Adequate tissue sample

Stratification

- Geographic region

- PD-L1 status

- Chemotherapy regimen
(B)

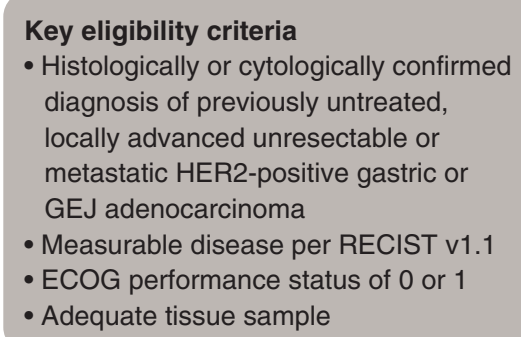

Stratification

-PD-L1 status
Pembrolizumab 200 mg Q3W + trastuzumab $8 \mathrm{mg} / \mathrm{kg}$ loading dose, and $6 \mathrm{mg} / \mathrm{kg}$ thereafter Q3W + investigator's choice of FP or CAPOX
Placebo (normal saline) Q3W + trastuzumab $8 \mathrm{mg} / \mathrm{kg}$ loading dose, and $6 \mathrm{mg} / \mathrm{kg}$ thereafter Q3W + investigator's choice of FP or CAPOX

Figure 1. KEYNOTE-811 study design. (A) Global cohort. (B) Japan-specific S-1 + oxaliplatin cohort. CAPOX: Capecitabine + oxaliplatin; FP: 5-Fluorouracil + cisplatin; R: Randomization; SOX: S-1 + oxaliplatin.

\section{Study design}

KEYNOTE-811 is an international, multicenter, randomized, double-blind, placebo-controlled, Phase III study consisting of two cohorts (Figure 1). The global cohort will receive pembrolizumab or placebo in combination with trastuzumab and either cisplatin + 5-fluorouracil (FP) or oxaliplatin + capecitabine (CAPOX); the Japanspecific cohort will receive pembrolizumab or placebo in combination with trastuzumab and S-1 + oxaliplatin (SOX). Eligible patients will be randomly assigned 1:1 to receive pembrolizumab $200 \mathrm{mg}$ or placebo (normal saline) + trastuzumab ( $8 \mathrm{mg} / \mathrm{kg}$ loading dose; $6 \mathrm{mg} / \mathrm{kg}$ thereafter) by intravenous infusion every 3 weeks and investigator's choice of FP or CAPOX in the global cohort or SOX in the Japan-specific cohort (Table 1). Treatment will continue until confirmed disease progression, unacceptable toxicity, investigator or patient decision to withdraw from the study, noncompliance with treatment or trial procedures, or completion of 35 cycles of study treatment ( $\sim 2$ years). Patients with progressive disease $(\mathrm{PD})$ whose conditions are clinically stable may continue on treatment at the discretion of the investigator. Patients who have attained CR may stop study treatment after receiving $\geq 8$ doses of pembrolizumab or placebo in total and $\geq 2$ doses after attaining confirmed CR. Patients with stable disease (SD) or better may be eligible for an additional 17 doses of pembrolizumab if their disease progresses while they are off study treatment provided they meet prespecified criteria.

Randomization will be performed centrally using an interactive voice/web response system. Pembrolizumab or placebo assignment will be masked in a double-blind fashion. Patients in the global cohort will be stratified by geographic region (Europe, Israel, North America and Australia vs Asia vs rest of world), PD-L1 status (positive $[\mathrm{CPS} \geq 1$ ] vs negative [CPS $<1]$ ), and chemotherapy regimen (FP vs CAPOX). Patients in the Japan-specific cohort will be stratified by PD-L1 status (positive vs negative). 
Table 1. Study treatments.

\begin{tabular}{|c|c|c|c|c|}
\hline Treatment & Dose & Frequency & Route of administration & Dosing time of each 3-week cycle \\
\hline Pembrolizumab & $200 \mathrm{mg}$ & Q3W & IV infusion & Day 1 of each cycle \\
\hline Placebo (normal saline) & NA & Q3W & IV infusion & Day 1 of each cycle \\
\hline \multicolumn{5}{|l|}{$\mathrm{FP}^{\dagger}$} \\
\hline - Cisplatin $\ddagger$ & $80 \mathrm{mg} / \mathrm{m}^{2} \mathbb{I}$ & Q3W & IV infusion & Day 1 of each cycle \\
\hline - 5-Fluorouracil & $800 \mathrm{mg} / \mathrm{m}^{2} /$ day $\mathbb{I}$ & Continuous & IV infusion & Days $1-5$ of each cycle \\
\hline \multicolumn{5}{|l|}{ CAPOX $^{\dagger}$} \\
\hline - Oxaliplatin & $130 \mathrm{mg} / \mathrm{m}^{2} \mathbb{I}$ & Q3W & IV infusion & Day 1 of each cycle \\
\hline - Capecitabine & $1000 \mathrm{mg} / \mathrm{m}^{2} \mathbb{I}$ & BID & Oral & Days $1-14$ of each cycle \\
\hline \multicolumn{5}{|l|}{ sox ${ }^{\S}$} \\
\hline$-\mathrm{S}-1$ & $\begin{array}{l}<1.25 \mathrm{~m}^{2} ; \mathrm{BSA}: 40 \mathrm{mg} \\
1.25 \text { to }<1.5 \mathrm{~m}^{2} ; \mathrm{BSA}: 50 \mathrm{mg} \\
\geq 1.5 \mathrm{~m}^{2} ; \mathrm{ISA}: 60 \mathrm{mg}\end{array}$ & BID & Oral & Days $1-14$ of each cycle \\
\hline - Oxaliplatin & $130 \mathrm{mg} / \mathrm{m}^{2} \mathbb{I}$ & Q3W & IV infusion & Day 1 of each cycle \\
\hline - Trastuzumab & $\begin{array}{l}8 \mathrm{mg} / \mathrm{kg} \text { loading dose } \\
6 \mathrm{mg} / \mathrm{kg} \text { maintenance dose }\end{array}$ & Q3W & IV infusion & Day 1 of each cycle \\
\hline \multicolumn{5}{|c|}{$\begin{array}{l}\text { † Choice of chemotherapy backbone to be decided by the investigator before randomization. } \\
\text { ‡Duration of cisplatin treatment may be capped at } 6 \text { cycles per local country guidelines; however, treatment with 5-fluorouracil may continue per protocol. } \\
\text { §SOX received only by patients in the Japan-specific cohort. } \\
\mathbb{I}_{\text {Body surface area to be calculated per local guidance. }} \\
\text { BID: Twice daily; CAPOX: Capecitabine + oxaliplatin; FP: 5-Fluorouracil + cisplatin; IV: Intravenous; Q3W: Every } 3 \text { weeks; SOX: S-1 + oxaliplatin. }\end{array}$} \\
\hline
\end{tabular}

\section{Eligibility criteria, planned sample size \& study period}

Eligibility criteria are described in detail in Table 2 . In brief, eligible patients are men or women aged $\geq 18$ years with a histologically or cytologically confirmed diagnosis of previously untreated, locally advanced unresectable or metastatic HER2-positive gastric or GEJ adenocarcinoma. The planned sample size is approximately 692 patients in the global cohort and 40 patients in the Japan-specific cohort, for a total of 732 patients. The estimated time after the first participant is randomly assigned to the time of the final analysis is approximately 56 months. The study started in October 2018, and the estimated completion date is 20 March 2024.

\section{Study end points}

The primary end points are PFS and OS. PFS is defined as the time from randomization to the first documented disease progression per Response Evaluation Criteria in Solid Tumors version 1.1 (RECIST v1.1) by blinded independent central review (BICR) or death from any cause, whichever occurs first. OS is defined as the time from randomization to death from any cause.

Secondary end points are ORR and DOR, per RECIST v1.1 by BICR and safety. DOR is defined as the time from first response (CR or PR) to subsequent disease progression or death from any cause, whichever occurs first.

Exploratory end points include health-related quality of life (HRQOL; assessed using the European Organization for the Research and Treatment of Cancer [EORTC] Quality of Life Questionnaire core 30 items [QLQ C30] and the gastric cancer module [STO22]), characterization of utilities (assessed using the EuroQol 5D 5-level [EQ5D-5L] questionnaire), molecular biomarkers and PFS and ORR per immune-related RECIST by investigator review.

\section{Study procedures}

Disease progression and tumor response will be assessed using computed tomography (CT) or, if CT is contraindicated, magnetic resonance imaging (MRI). Initial imaging will be performed within 28 days before randomization. The first on-study imaging assessment will be performed 6 weeks after randomization, and subsequent imaging will be performed every 6 weeks (or more frequently if clinically indicated). Imaging will continue until confirmation of progressive disease by BICR, initiation of new anticancer treatment, withdrawal of consent or death. Response will be confirmed by repeat imaging $\geq 4$ weeks after the first documentation of response. Patients who experience disease progression or start new anticancer therapy will be followed up for survival status by telephone every 12 weeks until death, withdrawal of consent, or end of study, whichever occurs first. 


\section{Table 2. Eligibility criteria.}

\section{Inclusion criteria}

\section{- Male or female}

- Age $\geq 18$ years

- Previously untreated histologically or cytologically confirmed locally advanced unresectable or metastatic gastric or GEJ adenocarcinoma

- HER2-positive disease, defined as either IHC $3+$ or IHC 2+ in combination with

$\mathrm{ISH}+$ (or FISH), as assessed by BICR on primary or metastatic tumor

- Measurable disease per RECIST v1.1 by site investigator

- ECOG PS 0 or 1

- Life expectancy $\geq 6$ months

- Willing to provide a tumor tissue sample adequate for PD-L1 and MSI biomarker analysis

- Adequate cardiac function, defined as left ventricular ejection fraction $\geq 55 \%$ as determined by MUGA scan or ECHO and QT interval calculated according to the

Fridericia method ( $\leq 470 \mathrm{~ms}$ for men and $\leq 480 \mathrm{~ms}$ for women)

- Adequate hematologic function, defined as ANC $\geq 1500 / \mu \mathrm{l}$, platelet count

$\geq 100,000 / \mu \mathrm{l}$ and hemoglobin $\geq 9.0 \mathrm{~g} / \mathrm{dl}$ or $\geq 5.6 \mathrm{mmol} / \mathrm{l}$

- Adequate renal function, defined as creatinine $\leq 1.5 \times$ ULN or measured or calculated creatinine clearance $>60 \mathrm{ml} / \mathrm{min}$ for those with creatinine levels $1.5 \times$ ULN

- Adequate hepatic function, defined as total bilirubin $\leq 1.5 \times$ ULN or direct bilirubin $\leq$ ULN for those with total bilirubin levels $>1.5 \times$ ULN, ALT/AST levels

$\leq 2.5 \times$ ULN or $\leq 5 \times$ ULN for those with liver metastases, and albumin $\geq 2.5 \mathrm{~g} / \mathrm{dl}$

- Adequate coagulation function, defined as INR $\leq 1.5 \times$ ULN, unless the patient is receiving anticoagulant therapy with $\mathrm{PT}$ or $\mathrm{APTT} / \mathrm{PTT}$ is within the therapeutic range

- Written informed consent
- Willing to use adequate contraception methods throughout the study and for 7

months after the last dose of study treatment

\section{Exclusion criteria}

- Previously received neoadjuvant or adjuvant therapy for locally advanced or metastatic disease (as long as it was completed $>6$ months before randomization without disease progression)

- Major surgery, open biopsy or significant traumatic injury $\leq \mathbf{2 8}$ days before randomization, or anticipated need for major surgery during the study treatment period

- Radiotherapy $\leq 14$ days of randomization

- Known additional malignancy that is progressing or has necessitated active treatment within the past 5 years (except BCC or SCC of the skin that has undergone potentially curative treatment or in situ cervical cancer)

- Known active CNS metastases and/or carcinomatous meningitis (patients with previously treated brain metastases may be eligible if disease is radiologically and clinically stable)

- Active autoimmune disease that has necessitated systemic treatment (other than replacement therapy) in the past 2 years

- Diagnosis of immunodeficiency or receiving long-term systemic steroid therapy ( $>10 \mathrm{mg}$ /day prednisone equivalent) or any other form of immunosuppression therapy within 7 days before the first dose of study treatment

- History of (noninfectious) pneumonitis treated with steroids or current pneumonitis

- History of active tuberculosis

- Active infection necessitating systemic therapy

- Poorly controlled diarrhea

- Accumulation of pleural, ascitic or pericardial fluid necessitating drainage or diuretic drugs $\leq 2$ weeks before enrollment

- History or current evidence of any condition, therapy, or laboratory abnormality that might confound the study results or interfere with study participation

- Peripheral neuropathy grade $>1$

- Psychiatric or substance abuse disorder that could impede cooperation with study requirements

- Positive urine pregnancy test $\leq 72 \mathrm{~h}$ before randomization (females of

childbearing potential)

- Pregnant or breastfeeding or expecting to conceive or father children within the projected study duration

- Active or clinically significant cardiac disease

- Known history of HIV, HBV or HCV infection

- Known hypersensitivity (grade $\geq 3$ ) to any of the study drugs or their excipients

- Active infection necessitating systemic therapy

- Allogeneic tissue or solid organ transplant

- Previous treatment with anti-PD-1, anti-PD-L1 or anti-PD-L2, or with an agent directed to another stimulatory or co-inhibitory T-cell receptor (e.g., CTLA4, OX40 and CD137)

- Immunized with live vaccine $\leq 30$ days before first dose of study treatment

- Participation in study of investigational agent or device $\leq 4$ weeks before the first dose of study treatment

ANC: Absolute neutrophil count; aPTT: Activated partial thromboplastin time; BCC: Basal cell carcinoma; BICR: Blinded-independent central review; CNS: Central nervous system; ECHO: Echocardiogram; ECOG: Eastern Cooperative Oncology Group; FISH: Fluorescent in-situ hybridization; GEJ: Gastroesophageal junction; HBV: Hepatitis B virus; HCV: Hepatitis C virus; HIV: Human immunodeficiency virus; IHC: Immunohistochemistry; INR: International normalized ratio; ISH: In-situ hybridization; MSI: Microsatellite instability; MUGA: Multigated acquisition; PT: Prothrombin time; PTT: Partial thromboplastin time; RECIST v1.1: Response Evaluation Criteria in Solid Tumors version 1.1; SCC: Squamous cell carcinoma; ULN: Upper limit of normal.

Safety will be monitored throughout the study and for 30 days after the end of treatment (90 days for serious adverse events and events of clinical interest). Safety analyses will include incidence, cause and outcome of adverse events and changes in vital signs and laboratory values. Adverse events will be assessed as defined by the National Cancer Institute Common Terminology Criteria for Adverse Events, version 4.0.

Patient-reported outcome assessments (QLQ-C30, QLQ-STO22 and EQ-5D-5L) will be completed by patients electronically at cycles 1, 2, 3, 4 and 5, and every 2 cycles thereafter up to 1 year or end of treatment, whichever comes first, and at the 30-day posttreatment follow-up visit. PD-L1 expression will be assessed using the PD-L1 IHC 22C3 pharmDx assay (Agilent Technologies, CA, USA) and measured using CPS. Additional biomarker investigation may include genomic, metabolic and proteomic analyses.

\section{Statistical analysis}

Efficacy will be assessed in the intent-to-treat population (all randomly assigned patients) by treatment group. Safety will be assessed in all randomly assigned patients who received $\geq 1$ dose of study treatment according to the treatment received. Primary hypotheses for PFS and OS will be evaluated by comparing pembrolizumab + trastuzumab and chemotherapy with placebo + trastuzumab and chemotherapy using a stratified log-rank test. The hazard ratio will 
be estimated using a stratified Cox regression model, and event rates over time will be estimated using the KaplanMeier method. ORR will be compared between treatment groups using the stratified Miettinen and Nurminen method. The overall Type I error over the primary end points (PFS and OS) and the key secondary end point (ORR) is strongly controlled at $2.5 \%$ (one-sided), with initially $0.2 \%$ allocated to ORR, $0.3 \%$ to PFS and $2 \%$ to OS.

For ORR, with a sample size of approximately 260 patients at the first interim analysis, the study has approximately $90 \%$ power for detecting a 25 -percentage point difference in ORR (73 vs 48\%) at an initially assigned 0.002 (onesided) significance level. For PFS, there will be approximately 606 events at the PFS final analysis; the study has approximately $95 \%$ power for detecting an HR of 0.7 at an initially assigned 0.003 (one-sided) significance level. For OS, there will be approximately 551 deaths at the OS final analysis; the study has approximately $90 \%$ power for detecting an HR of 0.75 at an initially assigned 0.020 (one-sided) significance level.

The Japan-specific cohort will be analyzed separately. Three interim analyses are planned based on projected enrollment and event accrual rates. An external data monitoring committee will review the results of the interim analyses to determine whether the study will continue per prespecified criteria.

\section{Conclusion}

There is promising evidence that pembrolizumab used in combination with trastuzumab + chemotherapy may provide significant clinical benefit with manageable toxicity in patients with advanced gastric cancer. Here we have described the methodology of the KEYNOTE-811 study, an ongoing Phase III trial designed to evaluate the efficacy and safety of pembrolizumab in combination with trastuzumab + chemotherapy as first-line treatment for patients with advanced HER2-positive gastric or GEJ adenocarcinoma. The study aims to show that the addition of pembrolizumab to a regimen of trastuzumab + chemotherapy will result in improved survival compared with treatment with trastuzumab + chemotherapy alone. It is hoped that the results of KEYNOTE-811 will further define the role of pembrolizumab and the feasibility of a dual antibody strategy in patients with advanced gastric cancer.

\section{Executive summary}

- Most patients with gastric cancer receive diagnoses at the advanced stage of the disease, when prognosis is poor.

- Given the limited survival benefit observed with currently recommended therapies, there remains a need for novel therapeutic regimens to treat patients with advanced gastric cancer.

Background \& rationale

- A growing body of evidence indicates that PD-1 and PD-L1 are frequently overexpressed in gastric cancer and that their upregulation may be prognostic of poor outcome.

- Pembrolizumab has shown durable clinical benefit in patients with advanced gastric cancer and is particularly promising when used in combination with chemotherapy.

- Combining trastuzumab and chemotherapy with pembrolizumab in the first-line setting might be beneficial for patients with HER2-positive disease.

KEYNOTE-811 study design \& eligibility criteria

- KEYNOTE-811 is an international, multicenter, randomized, double-blind, placebo-controlled, Phase III study evaluating the efficacy and safety of pembrolizumab in combination with trastuzumab and chemotherapy as first-line treatment of patients with advanced/metastatic HER2-positive gastric cancer.

- Eligible patients with a histologically or cytologically confirmed diagnosis of previously untreated, locally advanced unresectable or metastatic HER2-positive gastric or gastroesophageal junction adenocarcinoma will be randomly assigned to receive pembrolizumab or placebo in combination with trastuzumab and chemotherapy (cisplatin +5 -fluorouacil or oxaliplatin + capecitabine [S-1 + oxaliplatin will be given in the Japan-specific cohort]).

Outcome measures/end points

- The primary end points are progression-free survival and overall survival.

Conclusion

- It is hoped that the KEYNOTE-811 study will further define the role of pembrolizumab and the feasibility of a dual antibody strategy in patients with advanced gastric cancer. 
Supplementary data

An infographic accompanies this paper and is included at the end of the references section in the PDF version. To view or download this infographic in your browser please click here: www.futuremedicine.com/doi/suppl/10.2217/fon-2020-0737

Author contributions

Conception, design or planning of the study: HC Chung, YJ Bang, CS Fuchs, T Satoh, K Shitara, J Tabernero, E van Cutsem, ZA Cao, J Lu, P Bhagia, CS Shih and YY Janjigian. Acquisition of the study: HC Chung, YJ Bang, S-K Qin, T Satoh, K Shitara, M Alsina, J Lu, CS Shih and YY Janjigian. Analysis of the data: HC Chung, S-K Qin, T Satoh, CS Shih and YY Janjigian. Interpretation of the results: HC Chung, K Shitara, E van Cutsem, ZA Cao, CS Shih and YY Janjigian. Drafting of the manuscript: HC Chung, T Satoh, K Shitara, J Tabernero, J Lu, YY Janjigian. Critically reviewing or revising the manuscript for important intellectual content: HC Chung, YJ Bang, CS Fuchs, S-K Qin, T Satoh, K Shitara, J Tabernero, E van Cutsem, M Alsina, ZA Cao, P Bhagia, CS Shih and YY Janjigian. Final approval: all authors.

Acknowledgments

The authors thank the patients and their families and caregivers, as well as all investigators and site personnel, for participating in this trial.

Financial \& competing interests disclosure

Funding for this research was provided by Merck Sharp \& Dohme Corp., a subsidiary of Merck \& Co., Inc., NJ, USA. HC Chung reports grants to his institution and writing support from MSD during the conduct of the study. He also reports grants to his institution from Amgen, BeiGene, BMS/Ono, GSK, Lilly, Merck-Serono, MSD and Taiho; honoraria from Lilly and Merck Serono; and consultation fees from Amgen, BeiGene, BMS, Celltrion, Gloria, Lilly, Merck Serono, MS, Quintiles, Taiho and Zymework outside the submitted work. Y-J Bang served in a consulting/advisory role for Astellas, AstraZeneca, Bayer, BeiGene, BMS, Daiichi Sankyo, Eli Lilly, Genentech/Roche, Genexine, GreenCross, Hanmi, Merck Serono, MSD, Novartis, Samyang Biopharm and Taiho, and grants (to the institution for clinical trials) from Astellas, AstraZeneca, Bayer, BeiGene, Boehringer Ingelheim, Boston Biomedical, BMS, CKD Pharma, Curis, Daiichi Sankyo, Eli Lilly, Five Prime, Genentech/Roche, Genexine Green Cross, GSK, MacroGenics, Merck Serono, MSD, Novartis, Ono, Pfizer, Taiho and Takeda outside the submitted work. CS Fuchs served in an advisory/consultancy role for Agios, Amylin Pharmaceuticals, Bain Capital, CytomX Therapeutics, Daiichi Sankyo, Eli Lilly, Entrinsic Health, Evolvelmmune Therapeutics, Genentech, Merck, Taiho and Unum Therapeutics. He also serves as a director for CytomX Therapeutics and owns unexercised stock options for CytomX and Entrinsic Health. He is a co-founder of Evolvelmmune Therapeutics and has equity in this private company. S-K Qin reports no conflict of interest. T Satoh reports grants from Astellas, BMS, Chugai Pharmaceutical, Daiichi Sankyo, Eli Lilly, Gilead Sciences, MSD, Ono, Parexel, Taiho and Yakult Honsha; personal fees from Astellas, BMS, Chugai Pharmaceutical, Eli Lilly, MSD, Ono, Taiho, Takara Bio, Sanofi-Aventis and Yakult Honsha; and endorsed department from Chugai Pharmaceutical, Ono and Yakult Honsha. K Shitara served in an advisory role for AbbVie, Astellas, BMS, Eli Lilly, GSK, Merck Pharmaceutical, Novartis, Ono, Pfizer, Taiho and Takeda; received research funding from Astellas, Chugai Pharma, Dainippon Sumitomo Pharma, Daiichi Sankyo, Eli Lilly, Merck Pharmaceutical, Medi Science and Taiho and honoraria (lecture fees) from AbbVie, Novartis and Yakult. J Tabernero served in a scientific consultancy role for Array Biopharma, AstraZeneca, Bayer, BeiGene, Boehringer Ingelheim, Chugai, Genentech, Inc., Genmab A/S, Halozyme, Imugene Limited, Inflection Biosciences Limited, Ipsen, Kura Oncology, Lilly, MSD, Menarini, Merck Serono, Merrimack, Merus, Molecular Partners, Novartis, Peptomyc, Pfizer, Pharmacyclics, ProteoDesign SL, Rafael Pharmaceuticals, F. Hoffmann-La Roche Ltd, Sanofi, Seattle Genetics, Servier, Symphogen, Taiho, VCN Biosciences, Biocartis, Foundation Medicine, HalioDX SAS and Roche Diagnostics. E van Cutsem served in an advisory/consultancy role for Array, AstraZeneca, Bayer, Biocartis, Bristol Myers Squibb, Celgene, Daiichi Sankyo, Halozyme, GSK, Pierre Fabre, Incyte, Ipsen, Lilly, Merck Sharp \& Dohme Corp., a subsidiary of Merck \& Co., Inc., NJ, USA., Merck KGaA, Novartis, Pierre Fabre, Roche, Servier, Sirtex and Taiho and reports grants (to his institution) from Amgen, Bayer, Boehringer Ingelheim, Bristol Myers Squibb, Celgene, Ipsen, Lilly, Merck Sharp \& Dohme Corp., a subsidiary of Merck \& Co., Inc., NJ, USA., Merck KGaA, Novartis, Roche, and Servier. M Alsina reports honoraria from BMS, Lilly, MSD and Servier and served in an advisory/consultancy role for BMS, Lilly, MSD and Servier. ZA Cao reports employment at Merck Sharp \& Dohme Corp., a subsidiary of Merck \& Co., Inc., NJ, USA. J Lu reports employment at Merck Sharp \& Dohme Corp., a subsidiary of Merck \& Co., Inc., NJ, USA. Bhagia reports employment at Merck Sharp \& Dohme Corp., a subsidiary of Merck \& Co., Inc., NJ, USA. CS Shih reports employment at and is a stockholder of Merck Sharp \& Dohme Corp., a subsidiary of Merck \& Co., Inc., NJ, USA. YY Janjigian reports advisory fees from Bristol Myers Squibb, Lilly, Merck, Merck Serono and Pfizer; and research expenses from Amgen, Bayer, Boehringer Ingelheim, Genentech, Lilly and Roche. The authors have no other relevant affiliations or financial involvement with any organization or entity with a financial interest in or financial conflict with the subject matter or materials discussed in the manuscript apart from those disclosed. 
Medical writing and editorial assistance were provided by Holly C. Cappelli, PhD, CMPP, and Brian Szente, PhD, of ApotheCom (Yardley, PA, USA). This assistance was funded by Merck Sharp \& Dohme, a subsidiary of Merck \& Co, NJ, USA.

\section{Ethical conduct of research}

The authors attest that the study protocol was approved by the appropriate ethics committee or institutional review board at each participating center. The study was conducted in accordance with standards of Good Clinical Practice and the Declaration of Helsinki. All participants will provide written informed consent before enrollment.

\section{Data sharing statement}

Merck Sharp \& Dohme Corp., a subsidiary of Merck \& Co., Inc., NJ, USA's data sharing policy, including restrictions, is available at http://engagezone.msd.com/ds_documentation.php. Requests for access to the clinical study data can be submitted through the EngageZone site or via email to dataaccess@merck.com.

\section{Open access}

This work is licensed under the Attribution-NonCommercial-NoDerivatives 4.0 Unported License. To view a copy of this license, visit http://creativecommons.org/licenses/by-nc-nd/4.0/

\section{References}

Papers of special note have been highlighted as: $\bullet \bullet$ of considerable interest

1. Bray F, Ferlay J, Soerjomataram I, Siegel RL, Torre LA, Jemal A. Global cancer statistics 2018: GLOBOCAN estimates of incidence and mortality worldwide for 36 cancers in 185 countries. CA: Cancer J. Clin. 68(6), 394-424 (2018).

2. Colquhoun A, Arnold M, Ferlay J, Goodman KJ, Forman D, Soerjomataram I. Global patterns of cardia and non-cardia gastric cancer incidence in 2012. Gut 64(12), 1881-1888 (2015).

3. Plummer M, Franceschi S, Vignat J, Forman D, De Martel C. Global burden of gastric cancer attributable to Helicobacter pylori. Int. J. Cancer 136(2), 487-490 (2015).

4. The Cancer Genome Atlas Research Network. Comprehensive molecular characterization of gastric adenocarcinoma. Nature 513(7517), 202-209 (2014).

5. Sohn BH, Hwang JE, Jang HJ et al. Clinical significance of four molecular subtypes of gastric cancer identified by The Cancer Genome Atlas Project. Clin. Cancer Res. 23(15), doi:10.1158/1078-0432.Ccr-16-2211 (2017).

6. van Cutsem E, Bang YJ, Feng-Yi F et al. HER2 screening data from ToGA: targeting HER2 in gastric and gastroesophageal junction cancer. Gastric Cancer 18(3), 476-484 (2015).

7. Smyth EC, Verheij M, Allum W et al. Gastric cancer: ESMO clinical practice guidelines for diagnosis, treatment and follow-up. Ann. Oncol. 27(Suppl. 5), v38-v49 (2016).

8. van Cutsem E, Sagaert X, Topal B, Haustermans K, Prenen H. Gastric cancer. Lancet 388(10060), 2654-2664 (2016).

9. National Comprehensive Cancer Network. NCCN clinical practice guidelines in oncology (NCCN guidelines): gastric cancer (version 3.2020). (2020). https://www.nccn.org/professionals/physician_gls/pdf/gastric.pdf

10. Bang YJ, van Cutsem E, Feyereislova A et al. Trastuzumab in combination with chemotherapy versus chemotherapy alone for treatment of HER2-positive advanced gastric or gastro-oesophageal junction cancer (ToGA): a Phase 3, open-label, randomised controlled trial. Lancet 376(9742), 687-697 (2010).

11. Tabernero J, Hoff PM, Shen L et al. Pertuzumab plus trastuzumab and chemotherapy for HER2-positive metastatic gastric or gastro-oesophageal junction cancer (JACOB): final analysis of a double-blind, randomised, placebo-controlled Phase 3 study. Lancet Oncol. 19(10), 1372-1384 (2018).

12. Hecht JR, Bang YJ, Qin SK et al. Lapatinib in combination with capecitabine plus oxaliplatin in human epidermal growth factor receptor 2-positive advanced or metastatic gastric, esophageal, or gastroesophageal adenocarcinoma: TRIO-013/1OGiC - a randomized Phase III trial. J. Clin. Oncol. 34(5), 443-451 (2016).

13. Zhao D, Klempner SJ, Chao J. Progress and challenges in HER2-positive gastroesophageal adenocarcinoma. J. Hematol. Oncol. 12(1), 50 (2019).

14. Wilke $\mathrm{H}$, Muro K, van Cutsem E et al. Ramucirumab plus paclitaxel versus placebo plus paclitaxel in patients with previously treated advanced gastric or gastro-oesophageal junction adenocarcinoma (RAINBOW): a double-blind, randomised Phase 3 trial. Lancet Oncol. 15(11), 1224-1235 (2014).

15. Japanese Gastric Cancer Association. Japanese gastric cancer treatment guidelines 2018 (5th Ed). Gastric Cancer 24 1-21 (2021).

16. Alsaab HO, Sau S, Alzhrani R et al. PD-1 and PD-L1 checkpoint signaling inhibition for cancer immunotherapy: mechanism, combinations, and clinical outcome. Front. Pharmacol. 8, 561 (2017). 
17. Cui C, Yu B, Jiang Q, Li X, Shi K, Yang Z. The roles of PD-1/PD-L1 and its signalling pathway in gastrointestinal tract cancers. Clin. Exp. Pharmacol. Physiol. 46(1), 3-10 (2019).

18. Li J, Song-Hua B, Feng L et al. PD-1/PD-L1 antagonists in gastric cancer: current studies and perspectives. World J. Meta-Anal. 7(3), 101-109 (2019).

19. KEYTRUDA $^{\circledR}$ (pembrolizumab) injection, for intravenous use, 06/2020. Merck Sharp \& Dohme, Corp., NJ, USA (2020).

20. Muro K, Chung HC, Shankaran V et al. Pembrolizumab for patients with PD-L1-positive advanced gastric cancer (KEYNOTE-012): a multicentre, open-label, Phase 1b trial. Lancet Oncol. 17(6), 717-726 (2016).

-• Open-label Phase Ib study (KEYNOTE-012) reporting the safety and activity of pembrolizumab in patients with PD-L1-positive recurrent or metastatic adenocaricnoma of the stomach or gastroesophageal junction.

21. Fuchs CS, Doi T, Jang RW et al. Safety and efficacy of pembrolizumab monotherapy in patients with previously treated advanced gastric and gastroesophageal junction cancer: Phase 2 clinical KEYNOTE-059 trial. JAMA Oncol. 4(5), e180013 (2018).

-. Single-arm, Phase II study (KEYNOTE-059) evaluating the safety and efficacy of pembrolizumab in a cohort of patients with previously treated gastric or gastroesophageal junction cancer.

22. Shitara K, Ozguroglu M, Bang YJ et al. Pembrolizumab versus paclitaxel for previously treated, advanced gastric or gastro-oesophageal junction cancer (KEYNOTE-061): a randomised, open-label, controlled, Phase 3 trial. Lancet 392(10142), 123-133 (2018).

-• Randomized, open-label, controlled Phase III trial (KEYNOTE-061) comparing pembrolizumab with paclitaxel in patients with advanced gastric or gastroesophageal junction cancer that progressed on first-line chemotherapy.

23. Wainberg ZA, Fuchs CS, Tabernero J et al. Efficacy of pembrolizumab (pembro) monotherapy versus chemotherapy for PD-L1-positive (CPS $\geq 10$ ) advanced G/GEJ cancer in the Phase II KEYNOTE-059 (cohort 1) and Phase III KEYNOTE-061 and KEYNOTE-062 studies. J.Clin. Oncol. 38(Suppl. 4), 427 (2020).

24. Shitara K, van Cutsem E, Bang Y-S et al. Pembrolizumab or pembrolizumab plus chemotherapy versus chemotherapy for first-line, advanced gastric cancer (KEYNOTE-062): a randomised, controlled Phase 3 study. JAMA Oncol. 6(10), 1571-1580 (2020).

25. Bang YJ, Kang YK, Catenacci DV et al. Pembrolizumab alone or in combination with chemotherapy as first-line therapy for patients with advanced gastric or gastroesophageal junction adenocarcinoma: results from the Phase II nonrandomized KEYNOTE-059 study. Gastric Cancer 22(4), 828-837 (2019).

-• Multicohort, nonrandomized, Phase II study (KEYNOTE-059) evaluating first-line pembroliuzmab with or without chemotherapy in advanced gastric/gastroesophageal junction cancer in cohorts 2 and 3.

26. Krasniqi E, Barchiesi G, Pizzuti L et al. Immunotherapy in HER2-positive breast cancer: state of the art and future perspectives. J. Hematol. Oncol. 12(1), 111 (2019).

27. Chaganty BKR, Qiu S, Gest A et al. Trastuzumab upregulates PD-L1 as a potential mechanism of trastuzumab resistance through engagement of immune effector cells and stimulation of IFNgamma secretion. Cancer Lett. 430, 47-56 (2018).

28. Loi S, Giobbie-Hurder A, Gombos A et al. Pembrolizumab plus trastuzumab in trastuzumab-resistant, advanced, HER2-positive breast cancer (PANACEA): a single-arm, multicentre, Phase 1b/2 trial. Lancet Oncol 20(3), 371-382 (2019).

-. Single-arm Phase Ib/II trial (PANACEA) assessing the safety and antitumor activity of pembrolizumab added to trastuzumab in trastuzumab-resistant, advanced HER2-positive breast cancer.

29. Janjigian YY, Chou JF, Simmons M et al. First-line pembrolizumab (P), trastuzumab (T), capecitabine (C) and oxaliplatin $(\mathrm{O})$ in HER2-positive metastatic esophagogastric adenocarcinoma (mEGA). J. Clin. Oncol. 37(Suppl. 4), 62-62 (2019).

30. Janjigian YY, Maron SB, Chatila WK et al. First-line pembrolizumab and trastuzumab in HER2-positive oesophageal, gastric, or gastro-oesophageal junction cancer: an open-label, single-arm, Phase 2 trial. Lancet Oncol. 21(6), 821-831 (2020).

-• Phase II trial of assessing the safety and efficacy of first-line pembrolizumab, trastuzumab, capecitabine and oxaliplatin in HER2-positive metastatic esophagogastric adenocarcinoma.

31. Rha SY, Lee CK, Kim H. Targeting HER2 in combination with anti-PD-1 and chemotherapy confers a significant tumor shrinkage of gastric cancer: a multi-institutional Phase Ib/II trial of first-line triplet regimen (pembrolizumab, trastuzumab, chemotherapy) for HER2 positive advanced gastric cancer (AGC). J. Clin. Oncol. 38(Suppl. 4), 3081 (2020).

-. Multi-institutional Phase Ib/II trial (PANTHERA) of first-line triple combination (pembrolizumab, trastuzumab and chemotherapy) therapy for HER2-advanced gastric cancer. 
Pembrolizumab in combination with trastuzumab and chemotherapy for HER2-positive advanced gastric cancer: KEYNOTE-811

\section{Authors}

Hyun Cheol Chung, Yung-Jue Bang, Charles S. Fuchs, Shu-Kui Qin, Taroh Satoh, Kohe Shitara, Josep Tabernero, Eric Van Cutsem, Maria Alsina, Z. Alexander Cao, Jia Lu, Pooja Bhagia, Chie-Schin Shih \& Yelena Janjigian

\section{Article URL}

www.futuremedicine.com/doi/10.2217/fon-2020-0737

Trial registration number NCT03615326

\section{Objectives}

Primary

- Compare PFS between treatment groups

- Compare OS between treatment groups

Secondary

- Compare ORR per RECIST v1.1 by BICR between treatment groups

- Compare DOR per RECIST v1.1 by BICR between treatment groups

- Assess the safety and tolerability of pembrolizumab in combination with trastuzumab + chemotherapy

\section{Key eligibility criteria}

Histologically or cytologically confirmed diagnosis of previously untreated, locally advanced unresectable or metastatic HER2-positive gastric or GEJ adenocarcinoma

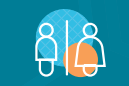

Men or women

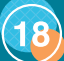

Age $\geq 18$ years

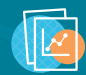

Measurable disease per RECIST v1.1

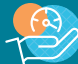

ECOG performance status of 0 or

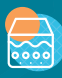

Adequate tissue sample

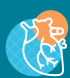

Adequate cardiac function defined as LVEF $>55 \%$ and QT interval

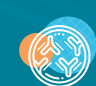

No immunodeficiency

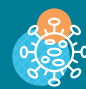

No active autoimmune disease

\section{Study design and treatment including planned sample size, planned study period and study procedures}

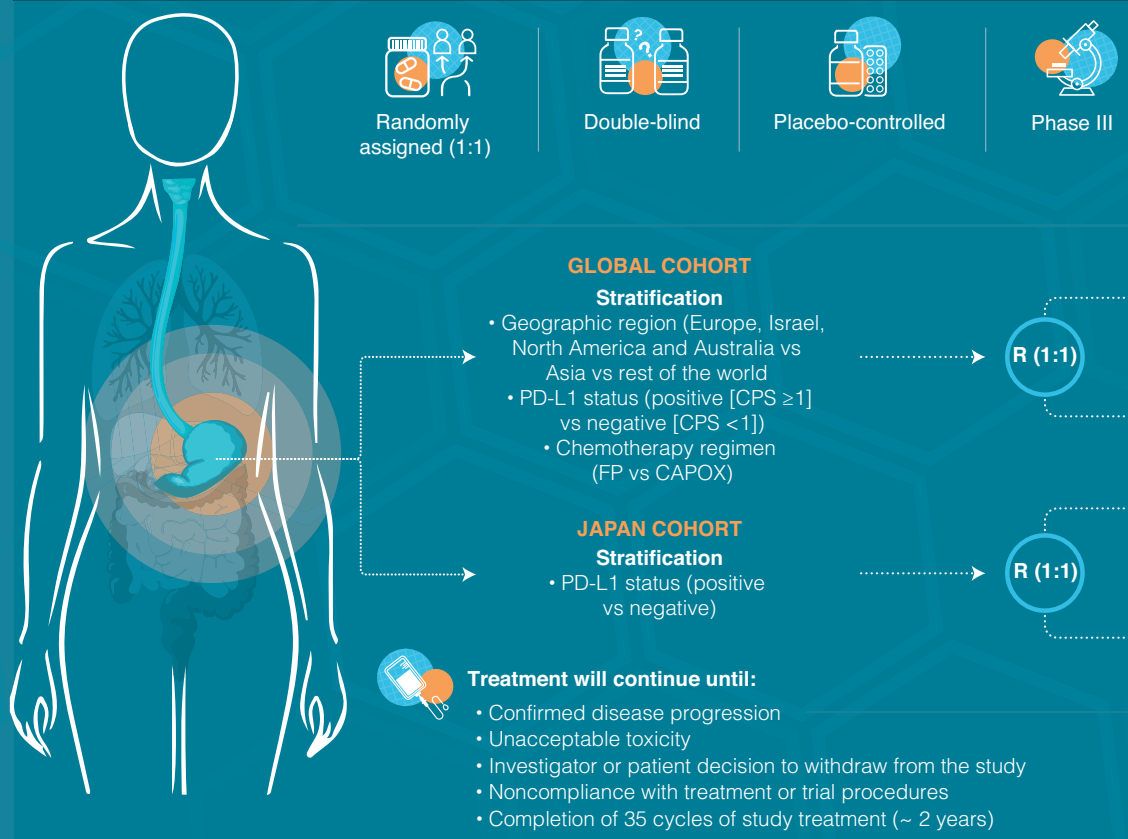

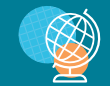

The planned sample size is $~ 692$ patients in the global cohor

$8 \mathrm{mg} / \mathrm{kg}$ loading dose, and $6 \mathrm{mg} / \mathrm{kg}$ thereafter + investigator's choice of FP or CAPOX Q3W

Placebo (normal saline) + trastuzumab $8 \mathrm{mg} / \mathrm{kg}$ loading dose, and $6 \mathrm{mg} / \mathrm{kg}$ thereafter + investigator's choice of FP or CAPOX Q3W

Pembrolizumab $200 \mathrm{mg}+$ trastuzumab $8 \mathrm{mg} / \mathrm{kg}$ loading dose, and $6 \mathrm{mg} / \mathrm{kg}$ thereafter + SOX Q3W

\section{Placebo (normal saline) $200 \mathrm{mg}+$
trastuzumab $8 \mathrm{mg} / \mathrm{kg}$ loading dose, and $6 \mathrm{mg} / \mathrm{kg}$ thereafter + SOX Q3W}

\section{Glossary}

BICR: Blinded independent central review; CAPOX: Oxaliplatin + capecitabine: CPS: Combined positive score; CR: Complete + capecitabine; CPS: Combined positve score; Ch. Comper response, DOR: Duraton of response, EORTC. Europe QLQ-C30: European Organization for the Research and QLQ-C30: European Organization for the Research and QLeatment of Cancer Qually of Life Questionnaire core 30 items Qreatment of Cancer Quality of Life Questionnesearch and cancer module: EQ-5D-5L: EuroQoL 5D 5-level: FP: Cisplatin + 5-fluouracil: GEJ: Gastric : Euphal 5D 5-level; FP: Cisplatin + 5. epidermal growh factor receptor 2, HRQOL: Healith-related Objective response rate: OS: Overall survivat; PDLL1: Objective response rate; OS: Overall survival; PD-L1:
Programmed death ligand 1; PFS: Progression-free survival: Pr: Partial respeath ligand 1; PFS: Progression-iree survival; in Solid Tumors version 1.1; SOX: S-1 + oxaliplatin

\section{Outcome measures/endpoints}

\section{Primary endpoints:}

- PFS (defined as the time from randomization to the first documented disease progression per RECIST $v 1.1$ by BICR or death from any cause, whichever occurs first) between treatment groups

- OS (defined as the time from randomization to death from any cause) between treatment groups

Secondary endpoints:

- ORR and DOR (defined as the time from first response [CR or PR] to subsequent disease progression or death from any cause, whichever occurs first), per RECIST v1.1 by BICR

- Safety

\section{Exploratory endpoints:}

- HRQOL (assessed using the EORTC QLQ-C30 and EORTC QLQ-STO22)

- Characterization of utilities (assessed using the EQ-5D-5L questionnaire)

- Molecular biomarkers

- PFS and ORR per immune-related RECIST by investigator review 